\title{
Excluding spatial sampling bias does not eliminate over-splitting in DNA-based species delimitation analyses
}

\author{
Daniel Lukic ${ }^{1}$, Jonas Eberle ${ }^{2}$, Jana Thormann ${ }^{1}$, Carolus Holzschuh ${ }^{3}$, and Dirk Ahrens ${ }^{1}$ \\ ${ }^{1}$ Zoological Research Museum Alexander Koenig \\ ${ }^{2}$ University of Salzburg \\ ${ }^{3}$ Affiliation not available
}

February 16, 2021

\begin{abstract}
DNA-barcoding and DNA-based species delimitation are major tools in DNA taxonomy. Sampling has been a central debate in this context, because the geographical composition of samples affect the accuracy and performance of DNA-barcoding. Performance of complex DNA-based species delimitation is to be tested under simpler conditions in absence of geographic sampling bias. Here, we present an empirical data set sampled from a single locality in a Southeast-Asian biodiversity hotspot (Laos: Phou Pan mountain). We investigate the performance of various species delimitation approaches on a megadiverse assemblage of herbivore chafer beetles (Coleoptera: Scarabaeidae) to infer whether species delimitation suffers in the same way from exaggerate infraspecific variation despite the lack of geographic genetic variation that led to inconsistencies between entities from DNA-based and morphology-based species inference in previous studies. For this purpose, a 658 bp fragment of the mitochondrial cytochrome c oxidase subunit 1 ( $\operatorname{cox} 1)$ was analysed for a total of 186 individuals of 56 morphospecies. Tree based and distance based species delimitation methods were used. All approaches showed a rather limited match ratio (max. 77\%) with morphospecies. PTP and TCS prevailingly over-splitted morphospecies, while 3\% clustering and ABGD also lumped several species into one entity. ABGD revealed the highest congruence between molecular operational taxonomic units (MOTUs) and morphospecies. Disagreements between morphospecies and MOTUs were discussed in the context of historically acquired geographic genetic differentiation, incomplete lineage sorting, and hybridization. The study once again highlights how important morphology still is in order to correctly interpret the results of molecular species delimitation.
\end{abstract}

\section{Excluding spatial sampling bias does not eliminate over-splitting in DNA-based species delim- itation analyses}

Daniel Lukic ${ }^{1}$, Jonas Eberle ${ }^{2}$, Jana Thormann ${ }^{1}$, Carolus Holzschuh ${ }^{3}$, Dirk Ahrens ${ }^{1,4}$

1 Zoologisches Forschungsmuseum Alexander Koenig, Zentrum fur Taxonomie und Evolutionsforschung, Adenauerallee 160, 53113 Bonn, Germany

${ }^{2}$ Paris-Lodron-Universität, Zoologische Evolutionsbiologie, Hellbrunner Straße 34, 5020 Salzburg, Austria

3 Spitzeckweg 11, 9500 Villach, Austria

4 corresponding author:d.ahrens@leibniz-zfmk.de; ahrens.dirk_col@gmx.de

\section{Abstract}

DNA-barcoding and DNA-based species delimitation are major tools in DNA taxonomy. Sampling has been a central debate in this context, because the geographical composition of samples affect the accuracy and performance of DNA-barcoding. Performance of complex DNA-based species delimitation is to be tested under simpler conditions in absence of geographic sampling bias. Here, we present an empirical data set sampled 
from a single locality in a Southeast-Asian biodiversity hotspot (Laos: Phou Pan mountain). We investigate the performance of various species delimitation approaches on a megadiverse assemblage of herbivore chafer beetles (Coleoptera: Scarabaeidae) to infer whether species delimitation suffers in the same way from exaggerate infraspecific variation despite the lack of geographic genetic variation that led to inconsistencies between entities from DNA-based and morphology-based species inference in previous studies. For this purpose, a $658 \mathrm{bp}$ fragment of the mitochondrial cytochrome c oxidase subunit 1 (cox1) was analysed for a total of 186 individuals of 56 morphospecies. Tree based and distance based species delimitation methods were used. All approaches showed a rather limited match ratio (max. 77\%) with morphospecies. PTP and TCS prevailingly over-splitted morphospecies, while 3\% clustering and ABGD also lumped several species into one entity. ABGD revealed the highest congruence between molecular operational taxonomic units (MOTUs) and morphospecies. Disagreements between morphospecies and MOTUs were discussed in the context of historically acquired geographic genetic differentiation, incomplete lineage sorting, and hybridization. The study once again highlights how important morphology still is in order to correctly interpret the results of molecular species delimitation.

\section{Key words}

Cox1, barcoding, species delimitation, geographic sampling bias, Laos.

\section{Introduction}

Since DNA barcoding was formally proposed on a large scale, cox1 sequences have been rapidly accumulated from all around the world (Porter \& Hajibabaei, 2018). Early studies mostly had a narrow systematic focus and targeted local or regional species assemblages. With emerging global comprehensiveness from the global iBOL project (International Barcode of Life), researchers became aware of the problems that arise with the use of cox1 (i.e., mitochondrial DNA) as taxonomic marker (Funk \& Omland 2003; Ballard \& Whitlock, 2004; Dasmahapatra \& Mallet, 2006; Dupuis et al., 2012; Nicholls et al., 2012; Smith et al., 2012; Dowton et al., 2014; Ross, 2014; Eberle et al., 2019), but also the effects of geographic scale on accuracy and performance of barcoding (Lohse, 2009; Bergsten et al., 2012; Gaytán et al., 2020). Therefore, geographic sampling has been a central debate (Lim et al., 2012; Reid \& Carstens, 2012; Talavera et al., 2013; Ahrens et al., 2016), in particular with respect to DNA barcoding, one of the major tools of DNA taxonomy.

In order to infer in more detail the nature of the genetic markers used for taxonomy, and also to investigate further the empirical behaviour of species delimitation approaches currently in use, it would be desirable to test commonly used methods on a dataset without geographic bias that still provides a sufficient number of closely related taxa. Here, we focus on $\operatorname{cox} 1$, since it continues and will continue to be a widely used marker for taxonomy in barcoding and metabarcoding studies.

So far, most comprehensive barcoding efforts have been made in "northern" and widely temperate countries (e.g., Pentinsaari et al., 2014, 2019; Gwiazdowski et al., 2015, Hendrich et al., 2015; Rougerie et al., 2015, Hebert et al., 2016, Rulik et al., 2017; Bouchard et al., 2017, Steinke et al., 2017; see also: ; https://www.bolgermany.de/wp/startseite/news-publikationen/publikationen/page/2/). The number of studies in tropical or subtropical areas is comparatively low or limited to a narrow focal group (e.g., Elias et al., 2007; Janzen et al., 2009; Janzen \& Hallwachs, 2011; Astrin et al., 2012; Ahrens et al., 2016; Cancian de Araujo et al., 2019), and only few authors assembled data on the global level (e.g., Zhou et al. 2016).

Interestingly, in regional (i.e., national) level libraries, molecular operational taxonomic units (MOTUs, i.e., BINs; Ratnasingham \& Hebert, 2013) showed perfect matches to known morphospecies in nearly $90 \%$ of the studied species (e.g., Pentinsaari et al., 2014; Hendrich et al., 2015). Occasionally, mismatch to described species occurred due to splitting into clusters of different geographic origin (e.g., Morinière et al., 2017) or sharing of identical or closely related haplotypes among different morphospecies (e.g., Hawlitschek et al., 2017). However, matches generally decreased when geographic sampling of species was wider, e.g., on a continental scale (Bergsten et al., 2012; Schmid-Egger et al., 2018, Mutanen et al., 2016), with 12-30\% 
of the species resulting paraphyletic. Identification success may decrease with increasing spatial scale of sampling; up to a drop of 50\% at continental scales (Bergsten et al., 2012). Sampling on a continental scale thus considerably increases the complexity of barcoding studies. Most of the "northern" latitude studies, however, are supposed to contain species with only low infraspecific haplotype diversity (due to extinctions and recolonization events during and after the Pleistocene; e.g., Hewitt, 1996, 1999; Schmitt, 2007; Ahrens et al., 2013), and often assemblages only contain a small number of closely related species. Thus, these data do not represent suitable test cases of species delimitation performance when the component of actual geographic genetic variation is excluded. On the other hand, several studies on tropical groups or locations also include specimens from one or more other sites (e.g., Elias et al., 2007; Thormann et al., 2016; Janzen et al., 2009; Janzen \& Hallwachs, 2011) or large amount of mismatch of MOTUs with morphospecies was seen as evidence for cryptic diversity (e.g., Janzen et al., 2009; Janzen \& Hallwachs, 2011).

Here we present a data set that was sampled from one local assemblage in a Southeast-Asian biodiversity hotspot (Laos: Phou Pan mountain). We investigate the performance of various species delimitation approaches on a megadiverse assemblage of herbivore chafer beetles (Coleoptera: Scarabaeidae: Sericini). Our objective is to infer whether species delimitation suffers from exaggerate infraspecific variation in the same way that led to inconsistencies between entities from DNA-based and morphology-based species inference in previous studies, despite the lack of geographic genetic variation. We are interested in the degree of deep coalescence in this local species assemblage and in how species delimitation approaches handle these data. Excluding geographic genetic variation we would expect less problems due to deep coalescences and thus higher rates of taxonomic congruence between morphospecies and MOTUs. Furthermore we employ clustering algorithms similar to those used in metabarcoding approaches, to explore the reliability of this critical step in current metabarcoding analyses pipelines (e.g., Coissac et al., 2012; Deiner et al., 2017; Macher et al., 2018; Ruppert et al., 2019).

\section{Material and methods}

\section{Study group, sampling and identification}

The study group is the megadiverse tribe Sericini that contains worldwide nearly 4000 described species in about 200 genera. They are one of the oldest lineages of phytophagous Scarabaeidae and diversified with the rise of the angiosperms $108 \mathrm{Ma}$. Sericini are nearly worldwide distributed, except in Australia, most oceanic islands, archipelagos, and circumpolar regions. The polyphagous herbivore adults are fully winged while larvae feed on roots and underground stems of living plants. Some species are considered as crop pests . Their highly similar external morphology makes the species difficult to distinguish, but highly complex male genitalia are well-differentiated between species and show only little intraspecific variation .

Sampling was conducted during four weeks in April, 2014 by Carolus Holzschuh and local collectors in the Phou Pan mountain area (Laos, Hua Phan province) (Fig. 1) (ca. $20^{\circ} 12^{\prime} \mathrm{N}, 104^{\circ} 01^{\prime} \mathrm{E}$ ), at an elevation between 1300 to 2000 meters. Specimens were collected using light traps, by hand, or netting during daytime. The Phou Pan mountain is situated in the Indo-Burmese biodiversity hotspot area (Myers et al., 2000) which is characterized by extremely high endemism. The habitat with its dense rainforests offers a large variety of plant species for herbivore insects to feed on. For this study we used only males (1086 specimens), since they were assignable to distinct morphospecies, while females are often not distinguishable among closely related syntopically occurring species. Samples were pinned after DNA extraction, dry mounted, labelled, and preserved at the ZFMK (Zoologisches Forschungsmuseum Alexander König, Bonn, Germany) (see Supplement Table 1).

Specimens were sorted to morphospecies by the complex shape of their copulation organ, i.e., aedeagus, which has been proven to be the best suited trait system to robustly infer the species entities for this group 
. For this purpose, male genitalia of all specimens were dissected. Habitus and genitalia of each species were photographed with a stereomicroscope (ZEISS Stereo Discovery.V20) connected to a ZEISS Axiocam. Presumably undescribed species that were not yet referable to an available species name, were numbered consecutively (sp1, sp2, etc.).

\section{DNA sequencing}

We sequenced the cox1 gene (5'-end) of multiple specimens (3-5) per morphospecies (in total 190). Lab work followed the standard protocols of the German Barcode of Life project. DNA was extracted from mesothoracic leg and attached muscles using the Qiagen DNeasy Blood and Tissue Kit (Hilden, Germany) or the Qiagen BioSprint96 magnetic bead extractor (Hilden, Germany).

The PCR reaction was carried out in total reaction mixes of $20 \mu \mathrm{l}$, including $2 \mu \mathrm{l}$ of undiluted DNA template, $0.8 \mu \mathrm{l}$ of each primer $(10 \mathrm{pmol} / \mu \mathrm{l})$, and standard amounts of the reagents provided with the "Multiplex PCR" kit from Qiagen (Hilden, Germany) using primers LCO1490-JJ [5'CHACWAAYCATAAAGATATYGG-3'] and HCO2198-JJ [5'-AWACTTCVGGRTGVCC AAARAATCA-3'] . Thermal cycling was performed on Applied Biosystems 2720 thermal cyclers (Life Technologies, Carlsbad, CA, USA), using a PCR program with two cycle sets, combining a "touchdown" and a "step-up" routine as follows: hot start Taq activation: 15 min at $95{ }^{\circ} \mathrm{C}$; first cycle set ( 15 repeats): $35 \mathrm{~s}$ denaturation at $94^{\circ} \mathrm{C}, 90 \mathrm{~s}$ annealing at $55^{\circ} \mathrm{C}(-1 \mathrm{deg} \mathrm{C}$ per cycle) and $90 \mathrm{~s}$ elongation at $72 \mathrm{degC}$; second cycle set (25 repeats): $35 \mathrm{~s}$ denaturation at $94 \mathrm{deg} \mathrm{C}, 90 \mathrm{~s}$ annealing at 40degC, and $90 \mathrm{~s}$ elongation at $72 \mathrm{degC}$; final elongation $10 \mathrm{~min}$ at $72 \mathrm{degC}$. Unpurified PCR products were subsequently sent for bidirectional Sanger sequencing to BGI Tech Solutions (Hongkong, China).

Raw DNA sequences were assembled (forward and reverse sequence) and edited in Geneious R7 (version 7.1.3, Biomatters Ltd.) to correct base-calling errors and to assign ambiguities (when forward and reverse sequence were not congruent for certain nucleotides). Sequences were aligned with Muscle (Edgar, 2004) as implemented into Geneious using the default settings. Primers were trimmed subsequently. All data are deposited in BOLD (project: SCOIB) and GenBank (accession numbers MW128167-MW128351) respectively (see Supplement Table 1).

\section{Phylogenetic analysis and species delimitation}

Putative morphospecies were compared with results obtained from the DNA-based species delimitation methods. We applied Poisson tree process (PTP), statistical parsimony network analysis (TCS), Automatic Barcode Gap Discovery (ABGD), distance based clustering, and Barcode of Life database (BOLD) - Barcode Index Numbers (BINs). These methods were applied on all sequenced beetles to result in clusters that are considered molecular taxonomic units (MOTUs), i.e., DNA-based species-assignments by the respective method.

A phylogenetic tree was calculated with maximum likelihood from the final multiple alignment of all DNA sequences using the IQ-TREE web server (IQ-TREE version 1.6.12; http://iqtree.cibiv.univie.ac.at/) ; the best substitution model (GTR+F+I+G4) was chosen with ModelFinder (Kalyaanamoorthy et al., 2017) according to Bayesian Information criterion (BIC). Branch support was calculated by generating 1000 samples for ultrafast bootstrapping (Hoang et al., 2018). The resulting tree was midpoint rooted in FigTree v1.4.3 (available from http://tree.bio.ed.ac.uk/software/figtree/). This tree was the basis for the PTP analysis. Additionally, split networks were generated using SplitsTree4 v. 4.16.1 to visualize incompatible and ambiguous signals in the cox1 dataset. In these networks the parallel edges, rather than the single branches, illustrate splits concluded from the data.

We used both versions of the Poisson tree process model (PTP) on the PTP web server (https://species.hits.org/; accessed on August 5th 2020): bPTP, which adds Bayesian support (pp) values to branches that 
delimit species in the input tree, and the refined multi-rate mPTP. PTP uses the shift in the number of substitutions at internal nodes to identify branching rate transition points which indicate speciation events. We used default settings for the bPTP analysis (100000 MCMC generations, thinning: 100, burn-in: 0.1, seed 123).

Statistical network analysis as performed with TCS v. 1.21 separates the sequence data into clusters of closely related haplotypes connected by changes that are non-homoplastic with a $95 \%$ probability (Templeton et al., 1992); if applied to mtDNA the extent of the networks has been found to be largely congruent with morphospecies

Automatic Barcode Gap Discovery (ABGD) was conducted using the ABGD webserver (https://bioinfo.mnhn.fr/abi/public/abgd/abgdweb.html; accessed on August 17th 2020) with default parameters (i.e., using Jukes-Cantor model (JC69) distances, a relative gap width of 1 and 50 steps, $\mathrm{Pmin}=0.001, \mathrm{Pmax}=0.1, \mathrm{Nb}$ bins for distance distribution=20). ABGD partitions individuals for a range of prior intraspecific distances, instead of using one predefined distance threshold . A robust result across a range of prior intraspecific distances was chosen as the best partition scheme. This outcome was also closest to the number of morphospecies and simultaneously matched the presumptive barcode gap in the histogram of distances.

Distance based clustering was done with the tclust-function in the R-package spider (v. 1.5.0; Brown et al., 2012). A threshold of $3 \%$ was applied to the pairwise distance matrix of all specimens that was corrected with the Kimura model (K80). The logic of this approach underlies most metabarcoding protocols (Macher et al., 2018; Piper et al., 2019; Liu et al., 2020), relying on the presence of a barcoding gap (Elbrecht et al., 2017), which was chosen as a gap at $3 \%$ pairwise distance by the majority of studies (however, see Beentjes et al., 2019 for an $2 \%$ example). Finally, we compared outcome from species delimitations to Barcode Index Number (BIN) assignments (Ratnasingham \& Hebert, 2013) in the BOLD data base (Project - SCOIB: Sericini COI Barcoding).

To check the performance and accuracy of the DNA-based delimitation methods compared to the a priori morphospecies hypotheses based on the genital morphology, the match ratio was calculated: Match ratio $=$ $2 * \mathrm{~N}_{\text {match }} /\left(\mathrm{N}_{\mathrm{MOTU}}+\mathrm{N}_{\text {morph }}\right) . \mathrm{N}_{\text {match }}$ is the number of species with exact matches, when the morphospecies and DNA-based species delimitation results to include the same specimens. $\mathrm{N}_{\text {MOTU }}$ is the number of classified groups by the different delimitation methods and finally $\mathrm{N}_{\text {morph }}$ is the number of morphospecies. All resulting MOTUs were mapped onto the phylogenetic tree beside terminal's labels (Fig. 2).

\section{Results}

\section{Morphospecies and sequencing success}

Fifty-six morphospecies could be determined from the Phu Phan mountain area. Twenty-seven of the morphospecies (48\%) were supposedly undescribed species or could not yet be assigned to an existing species (the fauna of mainland Asia has been fully revised in terms of type specimen revision (Ahrens, unpublished data), however, several species from Indonesia are known to widely occur in the Oriental region. Some species might thus still be assigned to already described species, when taxonomic revisions are finished for all parts of Asia).

186 specimens were sequenced successfully. The length of the alignedcox 1 sequences was 658 base pairs (bp). For Maladera sp 16 and Neoserica phuphanensis more than five individuals per morphospecies have been sequenced, since a few specimens were initially mistakenly assigned to other morphospecies. Of the 56 morphospecies 14 were singletons, i.e., only represented by one specimen per species. 
Due to shared haplotypes in different morphospecies, lowest inter- and infrasepcific distances were both zero (Table 2), while maximum infraspecific distances were around $7 \%$. The infraspecific mean distance was $1.5 \%$, and the median even lower $(0.8 \%)$. Nine morphospecies (i.e., $16 \%$ of the taxa) had infraspecific distances larger than $3 \%$.

\section{Species delimitation}

All morphospecies included in the analysis resulted monophyletic with three exceptions (Fig. 2): 1) $M i$ croserica sp 11 andMicroserica sp 13 are nested within the clade ofMicroserica varians ; 2) one of the five specimens ofNeoserica sp 29 was within the clade of Neoserica martinui ; and 3) Maladera sp 27 is placed within the clade of the morphologically very similar Maladera sp 9.

Three morphospecies shared identical haplotypes (Maladera sp 3a, sp 3b; sp 4; Figs 2, 3). Branch support values (ultrafast bootstrapping) of morphospecies clades are high with values of 0.8 to 1 . DNA-based species delimitation applying PTP, TCS, and ABGD resulted in different clusters. Thirty-one morphospecies showed congruent results for all DNA-based delimitation (Fig. 2). For 46 morphospecies the results of at least one method matched with the morphospecies assignment. All methods showed splitting and also lumping of morphospecies.

bPTP and mPTP subdivided the specimens into 70 and 65 MOTUs (Table 1), with 37 (bPTP) and 38 (mPTP) matches between the morphospecies and MOTUs. Deviations are caused by erroneously inferred splitting events (i.e., individuals of one morphospecies were separated into two or more different MOTUs). Match ratios of both PTP variants were relatively low: 0.59 and 0.63 , for bPTP and mPTP, respectively. TCS resulted in 69 MOTUs and had same number of matches (37) as bPTP. The match ratio (0.60) was higher than bPTP, but lower than mPTP.

ABGD yielded 51 MOTUs and showed the highest match ratio of all delimitation methods (0.77). It was the species delimitation method that showed most lumping of different morphospecies (Fig. 2). Examples for lumping are: one MOTU for Maladera exima plus M.parexima; Maladera sp 9 plus Ma. sp 27; Neoserica sp 37, N. martinui plus N. sp 29; as well as Microserica varians, Mi. sp 11 plus Mi. sp 13.

Distance based clustering at the $3 \%$ level yielded similar results to the previous methods. It found 62 MOTUs and matched with 40 morphospecies; the match ratio (0.68) was the second highest, after ABGD. Barcode Index Number (BIN) assignments revealed 65 MOTUs and matched as well with 40 morphospecies, however, its match ratio was lower $(0.66)$ than that of $3 \%$ clustering.

In $21 \%$ of the morphospecies $(n=12)$ we found relatively deep coalescence (i.e., distinct infraspecific phylogenetic structure) (e.g.,Ma. sp 4, sp 6, sp 16, Ma. fuscipes, Ma. futschauana, Ma. obscurata, Ma. peregoi , N. sp 26,Mi. panzona , Mi. varians , G. marginalis, G. carolusi ). In all others, infraspecific branches were rather shallow. Taxa sampled with more than three specimens and that were represented by a single haplotype did not occur. For all those cases with deep coalescence, at least one of the DNA-based species delimitations split the morphospecies, which in turn decreased the match ratio.

\section{Discussion}

In the present paper we investigated the DNA taxonomy of a megadiverse assemblage of chafer beetles (Sericini) with particular focus on the performance of commonly used species delimitation methods. The setup of examining barcodes of a single locality was chosen to investigate molecular species delimitation performance using data without geographic bias. While we know that match ratios strongly vary in tropical taxa (e.g., from 0.14 to 1.00; Ahrens et al., 2016), we theoretically expected that match ratios would go against one due to the exclusion of geography-induced variance. Instead, for different standard species delimitation methods, we could also not report match ratios higher than 0.77 . Interestingly, the $3 \%$ threshold clustering that is commonly used in metabarcoding approaches did not perform worse than more sophisticated 
approaches (like PTP, or TCS), however, an accuracy of only less than $80 \%$ is not really what one could call a reliable taxonomy assessment.

DNA-based species delimitation approaches may oversplit morphological entities (Ahrens et al., 2016), while at the same time the opposite may be also the case (Dalstein et al., 2019), even in the same taxon (as demonstrated here for the tribe Sericini). This particularly proved to be true in presence of incomplete lineage sorting and hybridisation and if geographic bias is not excluded (match ratio $<0.5$; Dalstein et al., 2019). Extreme over-splitting has been reported for both mtDNA and nDNA, when sex-biased dispersal occurs (Eberle et al., 2019) and were the general dispersal is in consequence also very limited.

Over-splitting in our data is caused by the relatively deep coalescence in $21 \%$ of the species, which widely corresponds with the missing match to the morphospecies, which is also reflected by the lack of a classical barcoding gap (Fig. 4). The impact is high with only 31 out of the 56 morphospecies matching perfectly the boundaries of inferred MOTUs (Fig. 2). The nature of maternal inheritance of mtDNA and its very low recombination rate is probably the major reason for these patterns of deep coalescence. Historically acquired genetic differentiation, for example in previously isolated populations, is maintained in secondarily mixing populations. The more often such isolated populations occur in time and space, for example due to climatic fluctuation during the Pleistocene in geographically highly structured areas such as Southeast Asia, the more often we encounter such "paleogeographically induced" infraspecific variation which leads to the same result as current geographic variation. This effect consequently impedes species delimitation methods in the same way, particularly in a single marker system (e.g., cox1 ).

Similarly, in our data we could also report cases of incomplete lineage sorting and/or hybridisation. In three cases, morphospecies were not monophyletic (Microserica sp 11/ Microserica sp 13 vs.Microserica varians ; Neoserica sp 29 vs. Neoserica martinui ; Maladera sp 27 vs. Maladera sp 9.), while another three morphospecies shared identical haplotypes (Maladerasp 3a, sp 3b; sp 4). In all cases, we may exclude cross contamination based on the position of the single samples on the DNA-extraction microtiter-plates. These cases do occur in only rather closely related species, which might show similar life traits (e.g., daytime activity inMicroserica), chemical communication, or mating behaviour (which is however, unknown for all species). In those instances, lumping of morphospecies in DNA based species delimitation seems to be more likely; however, also over-splitting was observed (e.g. Microserica). Despite strong divergence in male genital morphology, hybridization between closely related species of Sericini have been reported (e.g., . The rather divergent structure of the aedeagus of the different species might function with females by mechanical isolation (lock-and-key hypothesis) that prevents mating between different species. However, although there have been some recent work on the morphology of female genitalia, our knowledge on copulation functionality and mechanics is still not sufficient to tell if morphological structures of males and female genitalia actually function as a barrier, if only through tactile recognition by cryptic female choice .

Again, the present study demonstrates the necessity of an integrative taxonomy in the sense of Yeates et al. (2011) (see also . We showed that the use of different clustering- and tree-based delimitation methods (Carstens et al., 2013) with the same single maker reproduces the same erroneous signal in slightly different variations. It is thus critical to corroborate results with data from other sources (e.g., genital or larval morphology, feeding traits, behaviour, etc.; e.g. Janzen et al., 2009) to allow for independent testing of species boundaries.

Sericini chafers proved to be a valuable model system, because of robust morphospecies assignments that were facilitated by the highly dissimilar and morphologically complex male genitalia that perfectly serve as a species diagnostic trait .

Overall, the initial hypothesis of impeccable DNA-based species boundaries in syntopically co-occurring species assemblages clearly had to be rejected. This was rather unexpected, especially since there was no additional evidence from other sources that these over-splittings could relate to cryptic diversity (Janzen et al., 2009, 2017; Janzen \& Hallwachs, 2011).

Given the highly simplified parameters of DNA based species delimitation in this one-site species assemblage, 
it becomes clear how complex species delimitation with DNA-based methods is. Performance with mean error rates of more than $30 \%$ are under the expectations for proper use for applied sciences and conservation management. Even more sophisticated methods did not perform better than over-simplified threshold clustering methods as used for example in metabarcoding. Once more, we highlight the necessity of morphology for the verification ofde novo species delimitation results and the constant need of integrative taxonomic approaches.

\section{Acknowledgements}

We are grateful to Benedict Wipfler for his technical assistance. Furthermore, we are thankful to Silvia Fabrizi, Sasanka Ranasinghe, Julian Enss, Ralph Tollrian, Thomas Eltz and Hans-Joachim Krammer for helpful advice and discussions. This research was support by institutional funding of the ZFMK.

\section{References}

Ahrens, D. (2006). The phylogeny of Sericini and their position within the Scarabaeidae based on morphological characters (Coleoptera. Scarabaeidae). Systematic Entomology, 31 (1), 113-144. DOI: 10.1111/j.13653113.2005.00307.x.

Ahrens, D., Monaghan, M. T., \& Vogler, A. P. (2007). DNA-based taxonomy for associating adults and larvae in multi-species assemblages of chafers (Coleoptera. Scarabaeidae). Molecular Phylogenetics and Evolution , 44 (1), 436-449. DOI: 10.1016/j.ympev.2007.02.024.

Ahrens, D., \& Lago, P. K. (2008). Directional asymmetry reversal of male copulatory organs in chafer beetles (Coleoptera: Scarabaeidae): implications on left-right polarity determination in insect terminalia. Journal of Zoological Systematics and Evolutionary Research, 46 (2), 110-117. DOI: 10.1111/j.1439-0469.2007.00449.x.

Ahrens, D., Fabrizi, S., Sipek, P., \& Lago, P. (2013) Integrative analysis of DNA phylogeography and morphology of the European rose chafer (Cetonia aurata) to infer species taxonomy and patterns of postglacial colonisation in Europe. Molecular Phylogenetics and Evolution , 69: 83-94.

Ahrens, D., Schwarzer, J., \& Vogler, A. P. (2014). The evolution of scarab beetles tracks the sequential rise of angiosperms and mammals.Proceedings of the Royal Society B 281 (1791), p. 20141470. DOI: 10.1098/rspb.2014.1470.

Ahrens, D., Fujisawa, T., Krammer, H.-J., Eberle, J., Fabrizi, S., \& Vogler, A. P. (2016). Rarity and Incomplete Sampling in DNA-Based Species Delimitation. Systematic Biology , 65 (3), 478-494. DOI: 10.1093/sysbio/syw002.

Astrin, J. J., \& Stuben, P. E. (2008). Phylogeny in cryptic weevils: molecules, morphology and new genera of western Palaearctic Cryptorhynchinae (Coleoptera: Curculionidae). Invertebrate Systematics , 22 (5), 503-522. DOI: 10.1071/IS07057.

Avise, J. C. (2001). Phylogeography. The history and formation of species. 3. print. Cambridge, Mass.: Harvard Univ. Press.

Ballard, J.W.O. \& Whitlock, M.C. (2004) The incomplete natural history of mitochondria. Molecular Ecology , 13, 729-744

Barnard, A. A., Fincke, O. M., McPeek, M. A., \& Masly, J. P. (2017). Mechanical and tactile incompatibilities cause reproductive isolation between two young damselfly species. Evolution , 71 (10), 2410-2427. DOI: 10.1111/evo.13315. 
Beentjes, K.K., Speksnijder, A.G.C.L., Schilthuizen, M. , Hoogeveen, M., Pastoor, R., \& Hoorn, B.B. van der (2019) Increased performance of DNA metabarcoding of macroinvertebrates by taxonomic sorting. PLoS ONE 14(12), e0226527. https://doi.org/10.1371/journal.pone.0226527.

Bergsten, J., Bilton, D. T., Fujisawa, T., Elliott, M., Monaghan, M. T., Balke, M., ...\& Vogler, A. P. (2012). The effect of geographical scale of sampling on DNA barcoding. Systematic Biology , 61, 851-869. https ://doi.org/10.1093/sysbi o/sys037

Bond, J. E., \& Stockman, A. K. (2008). An integrative method for delimiting cohesion species: finding the population-species interface in a group of Californian trapdoor spiders with extreme genetic divergence and geographic structuring. Systematic Biology , 57 (4), 628-646. DOI: 10.1080/10635150802302443.

Bouchard, P., Smith, A.B.T., Douglas, H., Gimmel, M.L., Brunke, A.J., \& Kanda, K. (2017) Biodiversity of Coleoptera. In: Foottit, R.G., Adler, P.H. (Eds) Insect Biodiversity: Science and Society. John Wiley \& Sons Ltd, 337-417. https://doi.org/10.1002/9781444308211.ch11

Brown, S. D. J., Collins, R. A., Boyer, S., Lefort, M.-C., Malumbres-Olarte, J., Vink, C. J., \& Cruickshank, R. H. (2012). SPIDER: an R package for the analysis of species identity and evolution, with particular reference to DNA barcoding. Molecular Ecology Resources , 12, 562-565.

Cancian de Araujo, B., Schmidt, S., Schmidt, O., von Rintelen, T., von Rintelen, K., Floren, A., Ubaidillah, R., Peggie, D., Balke, M. (2019) DNA barcoding data release for Coleoptera from the Gunung Halimun canopy fogging workpackage of the Indonesian Biodiversity Information System (IndoBioSys) project. Biodiversity Data Journal , 7, e31432. https://doi.org/10.3897/BDJ.7.e31432

Carstens, B. C., Pelletier, T. A., Reid, N. M., Satler, J. D. (2013). How to fail at species delimitation. Molecular Ecology , 22 (17), 4369-4383. DOI: 10.1111/mec.12413.

Coissac, E., Riaz, T., Puillandre, N. (2012) Bioinformatic challenges for DNA metabarcoding of plants and animals. Molecular Ecology, 21, 1834e1847.

Dalstein, V., Eberle, J., Fabrizi, S., Etzbauer, C., Ahrens, D. (2019). COI-based species delimitation in Indochinese Tetraserica chafers reveal hybridisation despite strong divergence in male copulation organs. Organisms Diversity and Evolution , 19 (2), 277-286. DOI: 10.1007/s13127-019-00398-z.

Dasmahapatra, K.K. \& Mallet, J. (2006) Taxonomy: DNA barcodes: recent successes and future prospects. Heredity , 97, 254-255

Deiner, K., Bik, H.M., Machler, E., Seymour, M., Lacoursiere-Roussel, A., Altermatt, F., Creer, S., Bista, I., Lodge, D.M., de Vere, N., Pfrender, M.E., Bernatchez, L. (2017). Environmental DNA metabarcoding: Transforming how we survey animal and plant communities. Molecular Ecology , 26, 5872-5895.

Dowton, M., Meiklejohn, K., Cameron, S.L., Wallman, J. (2014) A preliminary framework for DNA barcoding, incorporating the multispecies coalescent. Systematic Biology , 63, 639-644

Dupuis, J.R., Roe, A.D., Sperling F. A. H. (2012) Multi-locus species delimitation in closely related animals and fungi: one marker is not enough. Molecular Ecology , 21, 4422-4436

Eberhard, W. G. (1985). Sexual Selection and Animal Genitalia. Cambridge, MA and London, England: Harvard University Press.

Eberle, J., Warnock, R. C. M., Ahrens, D. (2016). Bayesian species delimitation in Pleophylla chafers (Coleoptera) - the importance of prior choice and morphology. BMC Evolutionary Biology, 16, p. 94. DOI: 10.1186/s12862-016-0659-3.

Eberle, J., Fabrizi, S., Lago, P., Ahrens, D. (2017). A historical biogeography of megadiverse Sericini-another story "out of Africa"? Cladistics , 33 (2), 183-197. DOI: 10.1111/cla.12162. 
Eberle, J., Fabrizi, S., Bazzato, E., Rossi, Stella Columba, M., Cillo, D., Uliana M., Sparacio, I., Sabatinelli, G., Warnock, R.C.M., Carpaneto, G.M., Ahrens, D. (2019). Sex-Biased dispersal obscures species boundaries in integrative species delimitation approaches.Systematic Biology , 68, 441-459; published online (2018): doi: 10.1093/sysbio/syy072.

Edgar, R.C. (2004) MUSCLE: multiple sequence alignment with high accuracy and high throughput. Nucleic Acids Research, 32(5), 1792-97.

Elbrecht, V., Vamos, E. E., Meissner, K., Aroviita, J., \& Leese, F. (2017). Assessing strengths and weaknesses of DNA metabarcoding-based macroinvertebrate identification for routine stream monitoring. Methods in Ecology and Evolution , 8, 1265-1275. doi:10.1111/2041-210x.12789

Elias, M., Hill, R. I., Willmott, K. R., Dasmahapatra, Kanchon K., Brower, A. V. Z., Mallet, J., \& Jiggins, C. D. (2007). Limited performance of DNA barcoding in a diverse community of tropical butterflies. Proceedings of the Royal Society B , 274 (1627), 2881-2889. DOI: 10.1098/rspb.2007.1035.

Floyd R., Abebe E., Papert A., Blaxter M. (2002). Molecular barcodes for soil nematode identification. Molecular Ecology, 11 (4), 839-850. DOI: 10.1046/j.1365-294X.2002.01485.x.

Fontaneto, D., Flot, J.-F., Tang, C. Q. (2015). Guidelines for DNA taxonomy, with a focus on the meiofauna. Marine Biodiversity , 45 (3), 433-451. DOI: 10.1007/s12526-015-0319-7.

Funk, D.J. \& Omland, K.E. (2003) Species-level paraphyly and polyphyly: frequency, causes, and consequences, with insights from animal mitochondrial DNA. Annual Review of Ecology and Evolutionary Systematics , 34, 397-423

Gaytan, A, Bergsten, J, Canelo, T, Perez-Izquierdo, C, Santoro, M, Bonal, R. (2020) DNA Barcoding and geographical scale effect: The problems of undersampling genetic diversity hotspots. Ecology and Evolution , 10, 10754-10772. https://doi.org/10.1002/ece3.6733

Geiger, M. F., Moriniere, J., Hausmann, A., Haszprunar, G., Wagele, W., Hebert, P. D. N., Rulik, B. (2016). Testing the Global Malaise Trap Program - How well does the current barcode reference library identify flying insects in Germany? Biodiversity Data Journal , 4, e10671. DOI: 10.3897/BDJ.4.e10671.

Gwiazdowski, R.A., Foottit, R.G., Maw, H.E.L., Hebert, P.D.N., Imondi, R., \& La Rocca, G. (2015) The Hemiptera (Insecta) of Canada: Constructing a reference library of DNA barcodes. PLoS One, 10, e0125635. https://doi.org/10.1371/journal.pone.0125635

Hawlitschek, O., Moriniere, J., Lehmann, G.U.C., Lehmann, A.W., Kropf, M., Dunz, A., Glaw, F., Detcharoen, M., Schmidt, S., Hausmann, A., Szucsich, N.U., Caetano-Wyler, S.A., \& Haszprunar, G. (2017). DNA barcoding of crickets, katydids and grasshoppers (Orthoptera) from Central Europe with focus on Austria, Germany and Switzerland.Molecular Ecology Resources , 17, 1037-1053. doi:10.1111/1755-0998.12638

Hebert, P. D. N., Cywinska, A., Ball, Shelley L., \& deWaard, J. R. (2003a). Biological identifications through DNA barcodes.Proceedings of the Royal Society B , 270 (1512), 313-321. DOI: 10.1098/rspb.2002.2218.

Hebert, P. D. N., Ratnasingham, S., \& deWaard, J. R. (2003b). Barcoding animal life: cytochrome c oxidase subunit 1 divergences among closely related species. In Proceedings. Biological Letters, Suppl 270, S96-9. DOI: $10.1098 / \mathrm{rsbl} .2003 .0025$.

Hebert, P. D. N., Stoeckle, M. Y., Zemlak, T. S., \& Francis, C. M. (2004). Identification of Birds through DNA Barcodes. PLoS Biology , 2 (10), e312. DOI: 10.1371/journal.pbio.0020312.

Hebert, P. D. N., \& Gregory, T. R. (2005). The promise of DNA barcoding for taxonomy. Systematic Biology , 54 (5), 852-859. DOI: 10.1080/10635150500354886.

Hebert P. D. N., Ratnasingham S., Zakharov E.V., Telfer A.C., Levesque-Beaudin V., Milton M.A., Pedersen S., Jannetta P., \& DeWaard J.R. (2016) Counting animal species with DNA bar- 
codes: Canadian insects.Philosophical Transactions of the Royal Society of London B , 371, e1001127. https://doi.org/10.1098/rstb.2015.0333

Hewitt, G.M. (1996) Some genetic consequences of ice ages, and their role in divergence and speciation. Biological Journal of the Linnean Society , 58,247-276.

Hewitt, G.M. (1999) Post-glacial re-colonization of European biota.Biological Journal of the Linnean Society , 68, 87-112.

Hoang, D.T., Chernomor, O., Haeseler, A. von, Minh, B.Q., \& Vinh, L.S. (2018) UFBoot2: Improving the ultrafast bootstrap approximation.Molecular Biology and Evolution , 35:518-522. https://doi.org/10.1093/molbev/msx281

Huson, D. H., \& Bryant, D. (2006). Application of phylogenetic networks in evolutionary studies. Molecular Biology and Evolution, 23 (2), 254-267. DOI: 10.1093/molbev/msj030.

Janzen, D. H., Hallwachs, W., Blandin, P., et al. (2009). Integration of DNA barcoding into an ongoing inventory of complex tropical biodiversity. Molecular Ecology Resources , 9, Suppl s1, 1-26. doi:10.1111/j.17550998.2009.02628.x

Janzen, D. H., \& Hallwachs, W. (2011). Joining inventory by parataxonomists with DNA barcoding of a large complex tropical conserved wildland in northwestern Costa Rica. PLoS One, 6, e18123.

Janzen, D.H., Burns, J.M., Cong, Q., Hallwachs, W., Dapkey, T., Manjunath, R., Hajibabaei, M., Hebert, P.D.N., \& Grishin, N.V. (2017) Nuclear genomes distinguish cryptic species. Proceedings of the National Academy of Sciences , 114 (31), 8313-8318, DOI: 10.1073/pnas.1621504114

Joly, S., McLenachan, P. A., \& Lockhart, P. J. (2009). A statistical approach for distinguishing hybridization and incomplete lineage sorting. The American Naturalist, 174 (2), E54-70. DOI: 10.1086/600082.

Kalyaanamoorthy, S., Minh, B.Q., Wong, T.K.F., Haeseler, A. von, \& Jermiin, L.S. (2017) ModelFinder: Fast model selection for accurate phylogenetic estimates. Nature Methods , 14, 587-589. https://doi.org/10.1038/nmeth.4285

Knowles, L. L., Carstens, B. C. (2007). Delimiting species without monophyletic gene trees. Systematic Biology , 56 (6), 887-895. DOI: 10.1080/10635150701701091.

Liu, M., Clarke, L.J., Baker, S.C., Jordan, G.J., Burridge, C.P. (2020) A practical guide to DNA metabarcoding for entomological ecologists.Ecological Entomology , 45, 373-385.

Lohse, K. (2009). Can mtDNA barcodes be used to delimit species? A response to Pons et al. Systematic Biology , 58:439-442.

Macher, J.-N., Vivancos, A., Piggott, J.J., Centeno, F.C., Matthaei, C.D., \& Leese, F. (2018). Comparison of environmental DNA and bulk-sample metabarcoding using highly degenerate cytochrome c oxidase I primers. Molecular Ecology Resources , 18(6), 1456-1468 DOI 10.1111/1755-0998.12940.

Maddison, W. P. (1997). Gene Trees in Species Trees. Systematic Biology , 46 (3), 523-536. DOI: 10.1093/sysbio/46.3.523.

Meier, R., Shiyang, K., Vaidya, G., \& Ng, P. K. L. (2006). DNA barcoding and taxonomy in Diptera: a tale of high intraspecific variability and low identification success. Systematic Biology , 55 (5), 715-728. DOI: $10.1080 / 10635150600969864$.

Meyer, C. P., \& Paulay, G. (2005). DNA barcoding: error rates based on comprehensive sampling. PLoS Biology , 3 (12), e422. DOI: 10.1371/journal.pbio.0030422.

Miller, S. E. (2007). DNA barcoding and the renaissance of taxonomy.Proceedings of the National Academy of Sciences of the United States of America , 104 (12), 4775-4776. DOI: 10.1073/pnas.0700466104. 
Moriniere, J, Hendrich, L, Balke, M, et al. (2017). A DNA barcode library for Germany's mayflies, stoneflies and caddisflies (Ephemeroptera, Plecoptera and Trichoptera). Molecular Ecology Resources, 17, 1293- 1307. https://doi.org/10.1111/1755-0998.12683

Moritz, C., Cicero, C. (2004). DNA barcoding. Promise and pitfalls.PLoS Biology , 2 (10), e354. DOI: 10.1371/journal.pbio.0020354.

Muller, D., Suess, S., Hoffmann, A. A., Buchholz, G. (2013). The Value of Satellite-Based Active Fire Data for Monitoring, Reporting and Verification of REDD+ in the Lao PDR. Human Ecology, 41 (1), 7-20. DOI: 10.1007/s10745-013-9565-0.

Mutanen, M., Kivela, S.M., Vos, R.A., et al. (2016). Species-Level Para- and Polyphyly in DNA Barcode Gene Trees: Strong Operational Bias in European Lepidoptera. Systematic Biology , 65(6), 1024-1040. doi:10.1093/sysbio/syw044

Nair, M. R. G. K. (1986). Insects and mites of crops in India. (Ed. 2). Indian Council of Agricultural Research, New Delhi, 1975, 404pp.

Nguyen, L.-T., Schmidt, H.A., Haeseler, A. von, \& Minh, B.Q. (2015). IQ-TREE: A fast and effective stochastic algorithm for estimating maximum likelihood phylogenies. Molecular Biology and Evolution , 32, 268-274. https://doi.org/10.1093/molbev/msu300

Nicholls, J.A. et al. (2012). Mitochondrial barcodes are diagnostic of shared refugia but not species in hybridizing oak gall wasps. Molecular Ecology , 21, 4051-4062.

Ozgul-Siemund, A., \& Ahrens, D. (2015). Taxonomic utility of female copulation organs in Sericini chafers (Coleoptera, Scarabaeidae), with special reference to asymmetry. Contributions to Zoology , 84 (2), 167-178. DOI: 10.1163/18759866-08402005.

Padial, J. M., Miralles, A., La Riva, I. de, Vences, M. (2010). The integrative future of taxonomy. Frontiers in Zoology , 7 (1), 16. DOI: 10.1186/1742-9994-7-16.

Pentinsaari, M., Hebert, P.D.N., \& Mutanen, M. (2014). Barcoding Beetles: A regional survey of 1872 species reveals high identification success and unusually deep interspecific divergences. PLoS One , 9, e108651. https://doi.org/10.1371/journal.pone.0108651

Pentinsaari, M., Anderson, R., Borowiec, L., Bouchard, P., Brunke, A., Douglas, H., Smith, A.B.T., Hebert, P.D.N. (2019). DNA barcodes reveal 63 overlooked species of Canadian beetles (Insecta, Coleoptera).ZooKeys , 894, 53-150.

Piper, A.M., Batovska, J., Cogan, N.O.I, Weiss, J., Cunningham, J.P., Rodoni, B.C., Blacket, M.J. (2019) Prospects and challenges of implementing DNA metabarcoding for high-throughput insect surveillance. Giga Science, , 8, 1-22.

Porter, T.M., Hajibabaei, M. (2018) Over 2.5 million COI sequences in GenBank and growing. PLoS One , 13(9),e0200177. https://doi.org/10.1371/journal.pone.0200177 PMID: 30192752

Puillandre, N., Lambert, A., Brouillet, S., Achaz, G. (2012). ABGD, Automatic Barcode Gap Discovery for primary species delimitation. Molecular Ecology , 21 (8), 1864-1877. DOI: 10.1111/j.1365-294X.2011.05239.x.

Ratnasingham, S., \& Hebert, P. D. N. (2007). BOLD: The Barcode of Life Data System (http://www.barcodinglife.org). Molecular Ecology Notes , 7 (3), 355-364. DOI: 10.1111/j.14718286.2007.01678.x.

Ratnasingham, S., Hebert, P.D.N. (2013). A DNA-based registry for all animal species: the Barcode Index Number (BIN) system. PLoS One, 8, e66213.

Reid, N.M., \& Carstens, B.C. 2012. Phylogenetic estimation error can decrease the accuracy of species delimitation: a Bayesian implementation of the general mixed Yule-coalescent model. BMC Evolutionary 
Biology, 12:196.

Ritcher, P. O. (1966). White grubs and their allies. A Study of North American Scarabaeoid Larvae. Oregon State Monographs. Studies in Entomology , 4. Corvallis, Oregon State University Press, 219pp.

Ross, H.A. (2014) The incidence of species-level paraphyly in animals: a re-assessment. Molecular Phylogenetics and Evolution , 76, 10-17

Rougerie, R., Lopez-Vaamonde, C., Barnouin, T., Delnatte, J., Moulin, N., Noblecourt, T., Nusillard, B., Parmain, G., Soldati, F., \& Bouget, C. (2015) PASSIFOR: A reference library of DNA barcodes for French saproxylic beetles (Insecta, Coleoptera). Biodiversity Data Journal , 3, e4078. https://doi.org/10.3897/BDJ.3.e4078

Ruppert, K.M., Kline, R.J., Rahman, M.S. (2019) Past, present, and future perspectives of environmental DNA (eDNA) metabarcoding: A systematic review in methods, monitoring, and applications of global eDNA. Global Ecology and Conservation, 17, e00547.

Schmid-Egger, C., Straka, J., Ljubomirov, T., Blagoev, G.A., Moriniere, J., Schmidt, S. (2019). DNA barcodes identify 99 percent of apoid wasp species (Hymenoptera: Ampulicidae, Crabronidae, Sphecidae) from the Western Palearctic. Molecular Ecology Resources , 19, 476- 484. https://doi.org/10.1111/17550998.12963

Schmitt, T. (2007) Molecular biogeography of Europe: Pleistocene cycles and postglacial trends. Frontiers in Zoology , 4, 11. doi:10.1186/1742-9994-4-11

Smith, M.A. et al. (2012) Wolbachia and DNA barcoding insects: patterns, potential, and problems. PLoS One 7 , e36514

Steinke, D., Breton, V., Berzitis, E., Hebert, P.D.N. (2017) The School Malaise Trap Program: Coupling educational outreach with scientific discovery. PLoS Biology , 15, e2001829. https://doi.org/10.1371/journal.pbio.2001829

Talavera, G., Dinca, V., \& Vila, R. (2013). Factors affecting species delimitations with the GMYC model: insights from a butterfly survey. Methods in Ecology and Evolution, 4, 1101-1110.

Templeton, A. R., Crandall, K. A., Sing, C. F. (1992). A cladistic analysis of phenotypic associations with haplotypes inferred from restriction endonuclease mapping and DNA sequence data. III. Cladogram estimation. Genetics , 132 (2), 619-633.

Thormann, B., Ahrens, D., Marin Armijos, D., Peters, M.K., Wagner, T., Wagele, J.W. (2016) Exploring the Leaf Beetle Fauna (Coleoptera: Chrysomelidae) of an Ecuadorian Mountain Forest Using DNA Barcoding.PLoS One, 11(2), e0148268. doi:10.1371/journal.pone.0148268.

Trifinopoulos, J., Nguyen, L.-T., Haeseler, A. von, \& Minh, B. Q. (2016). W-IQ-TREE: a fast online phylogenetic tool for maximum likelihood analysis. Nucleic Acids Research , 44 (W1), W232-5. DOI: $10.1093 / \mathrm{nar} / \mathrm{gkw} 256$.

Yeates, D. K., Seago, A., Nelson, L., Cameron, Stephen L., Joseph, L. E.O., Trueman, J. W. H. (2011). Integrative taxonomy, or iterative taxonomy? Systematic Entomology, 36 (2), 209-217. DOI: 10.1111/j.13653113.2010.00558.x.

Zhang, J., Kapli, P., Pavlidis, P., Stamatakis, A. (2013). A general species delimitation method with applications to phylogenetic placements. In Bioinformatics 29 (22), 2869-2876. DOI: 10.1093/bioinformatics/btt499.

Zhou, X. et al. (2016). The Trichoptera barcode initiative: a strategy for generating a species-level Tree of Life. Phil. Trans. R. Soc.B, 371, 20160025. http://dx.doi.org/10.1098/rstb.2016.0025

Data Accessibility: 
- DNA sequences: Genbank accessions (MW128167-MW128351);

- DNA sequences (incl. abi files, locality data): BARCODE OF LIFE DATA SYSTEM (https://doi.org/10.5883/DS-DS-SCOIBL.\%0d\%0d\%20)

Table 1. Match ratio (after of DNA-based species delimitation methods on Sericini chafer data based on number of MOTUs and number of matches between MOTUs and morphospecies $\left(\mathrm{N}_{\text {morph }}=56\right)$.

\begin{tabular}{lllllll}
\hline & bPTP & mPTP & TCS & ABGD & 3\%Clust & BINs \\
\hline $\mathbf{N}_{\text {matches }}$ & 37 & 38 & 37 & 41 & 40 & 40 \\
$\mathbf{N}_{\text {MOTU }}$ & 70 & 65 & 68 & 51 & 62 & 65 \\
Match ratio & 0.59 & 0.63 & 0.60 & 0.77 & 0.68 & 0.66 \\
\hline
\end{tabular}

Table 2. Infra-and interspecific genetic distances of thecox1 dataset based on morphospecies assignments, as well as number of cases beyond an arbitral 3\% threshold distance being often used for MOTU clustering in Metabarcoding studies.

\begin{tabular}{lllll}
\hline & interspecific & interspecific & infraspecific & infraspecific \\
\hline & $\mathbf{k} \mathbf{2} \mathbf{p}$ & $\mathbf{m l}$ & $\mathbf{k 2 p}$ & $\mathbf{m l}$ \\
$\mathbf{m i n}$ & 0 & 0 & 0 & 0 \\
$\mathbf{m a x}$ & 0.24 & 0.24 & 0.074 & 0.073 \\
mean & 0.17 & 0.18 & 0.015 & 0.015 \\
median & 0.17 & 0.18 & 0.0083 & 0.0083 \\
$\mathbf{N}_{\text {dist }>\mathbf{3 \%}}$ & - & - & 9 & 9 \\
$\mathbf{N}_{\text {dist }<\mathbf{3 \%}}$ & 9 & 9 & - & - \\
\hline
\end{tabular}

Fig. 1. Collecting area in Laos $\left(20^{\circ} 12^{\prime} \mathrm{N}, 104^{\circ} 01^{\prime} \mathrm{E}\right)$ (marked with a black dot).

Fig. 2. Rooted maximum likelihood tree with information about morphospecies assignments, results of species delimitations (bPTP, mPTP, TCS, ABGD, BOLD, and BINs) and photographs of the aedeagi (lateral view). Green boxes indicate agreement between molecular species delimitation method and morphospecies assignment, while red boxes indicate disagreement. Ultra fast bootstrap supports $>0.5$ is shown above nodes. Genus name abbreviations: Chr. -Chrysoserica, G. - Gastroserica, Ma. - Maladera, Mi. - Microserica, N. - Neoserica, L. -Lasioserica, S. - Serica .

Fig. 3. Split network of all examined specimens. Singletons are highlighted in blue squares, others in orange colours. Morphospecies nested within others are highlighted with red squares or circles around them.

Fig. 4. Frequency of intra-V and interspecific distances of the Sericini data from Mt. Phu Pan (Laos). 


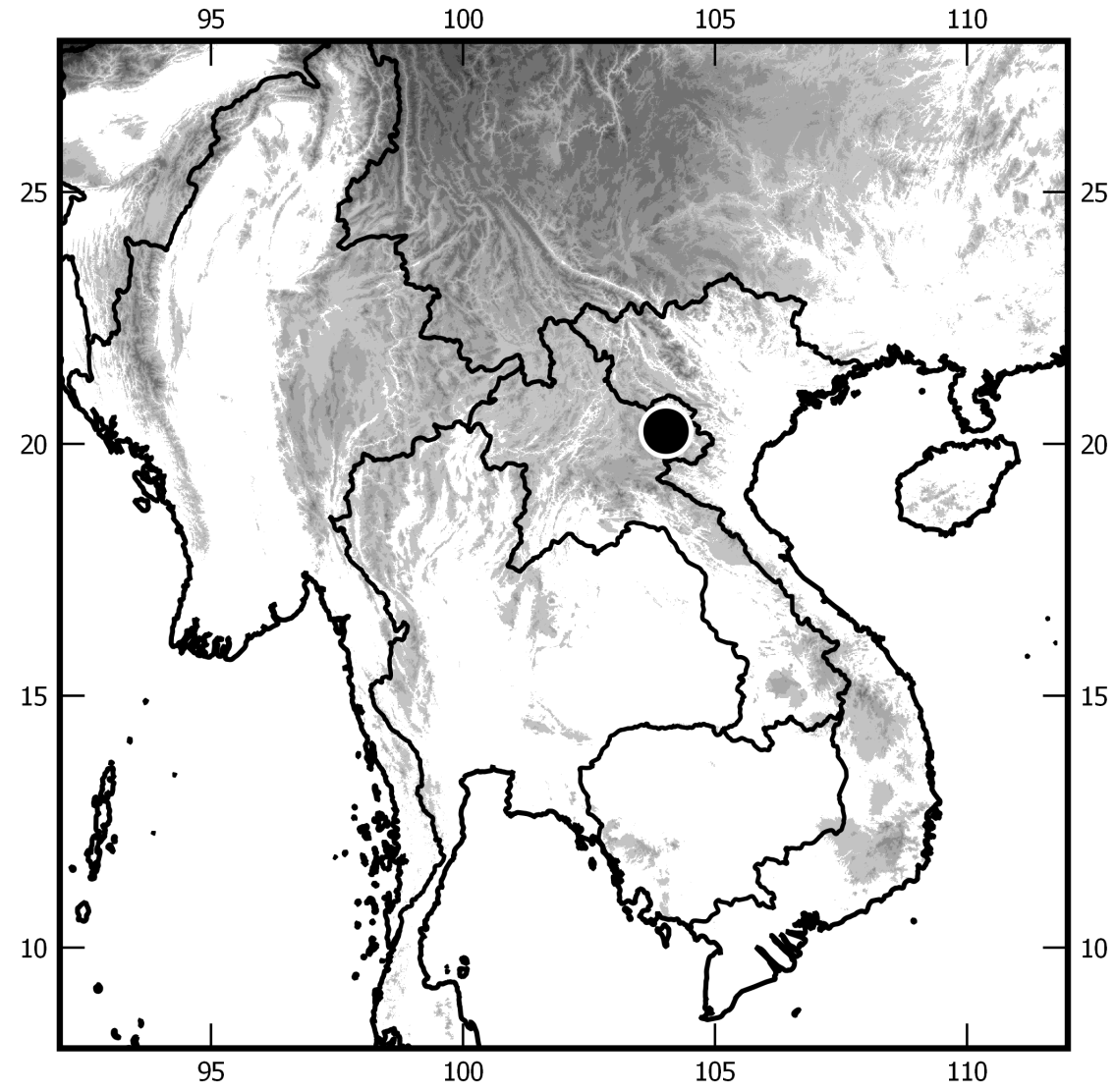




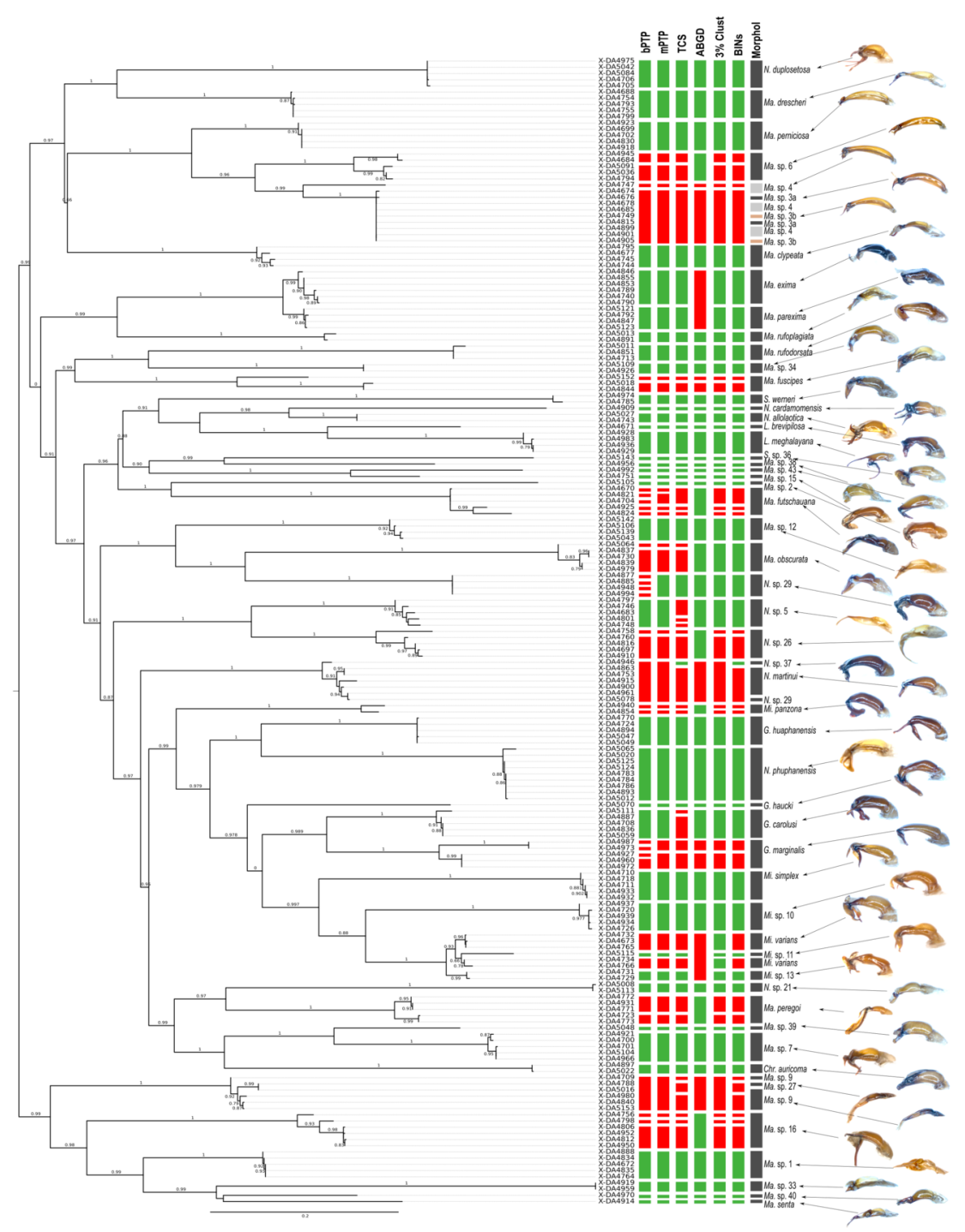



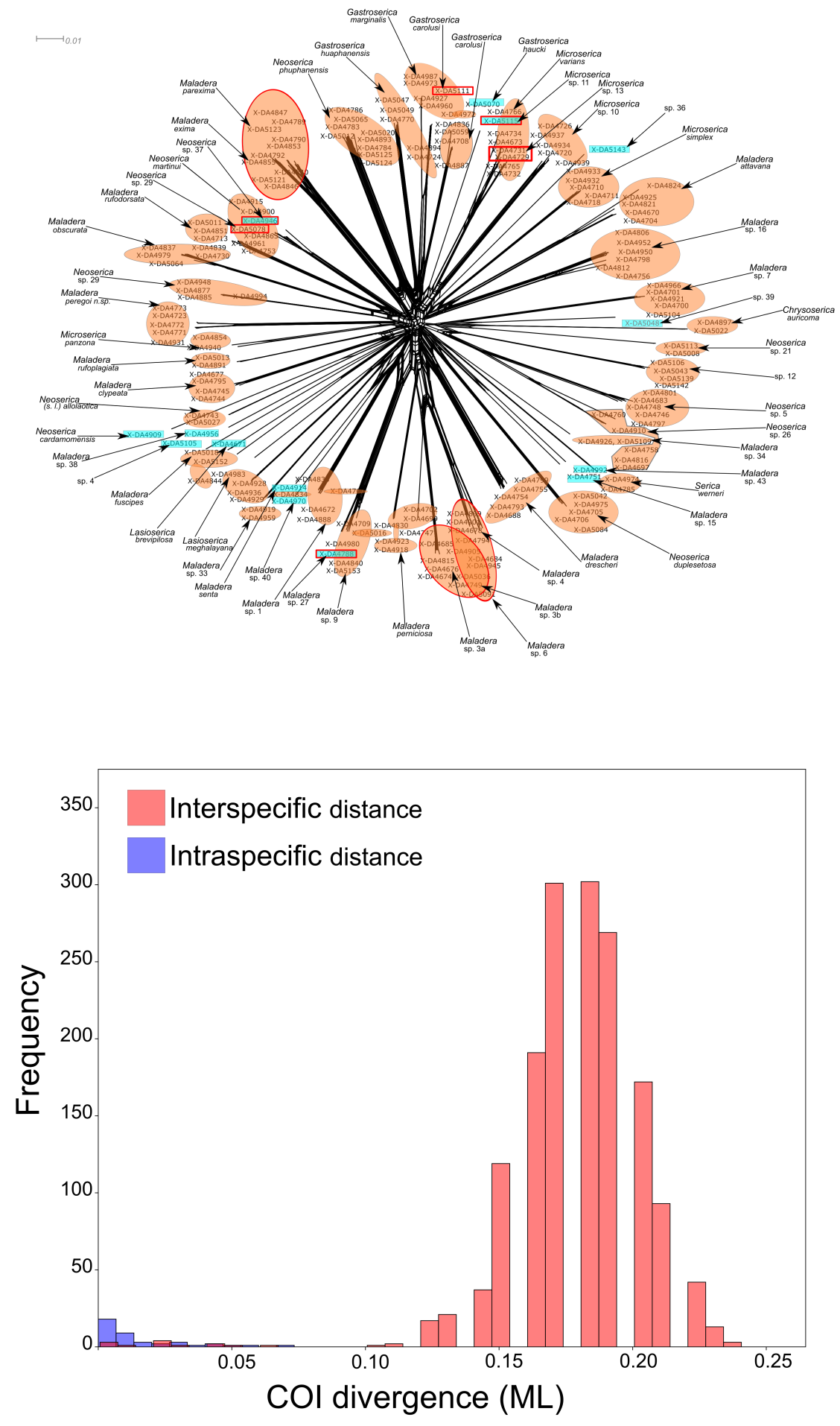